\title{
Angewandte
}

Supporting Information

(C) Wiley-VCH 2010

69451 Weinheim, Germany

\section{Monolithic and Flexible Polyimide Film Microreactors for Organic Microchemical Applications Fabricated by Laser Ablation**}

Kyoung-Ik Min, Tae-Ho Lee, Chan Pil Park, Zhi-Yong Wu, Hubert H. Girault, Ilhyong Ryu, Takahide Fukuyama, Yu Mukai, and Dong-Pyo Kim*

anie_201002004_sm_miscellaneous_information.pdf 
General. Both PI films as a chip substrate (Kapton ${ }^{\circledR} \mathrm{HN}$ film, $125 \mu \mathrm{m}$ ) and as a bonding layer (Kapton ${ }^{\circledR}$ EKJ film, $\left.50 \mu \mathrm{m}\right)$ were purchased from Dupont, USA. The laser ablation of the polyimide film was conducted by ArF excimer laser (193 nm, Lambda physik, Germany) or UV laser (355 nm, ESI, USA). For organic reactions, all chemicals were purchased from Sigma-Aldrich and used without additional purification. All liquid samples were pressure-driven into the channel using syringe pumps (KDS 100, KD Scientific Inc., Holliston, MA, USA) in the range of flow rates. The quantitative analysis of products was performed with a GC-MS (Gas chromatographymass analyzer, HP $5890 \mathrm{GC}$ and $5972 \mathrm{MSD}$, USA) with a capillary column (DB-35MS, $0.25 \mu \mathrm{m} \times 30 \mathrm{~m} \times 0.32 \mathrm{~mm}$ ), and also a NMR (Nuclear magnetic resonance, JEOL JMN-500 (500 MHz), Japan).

Fabrication of Microchannel. For T-shaped microchannels, a metal mask used to define the laser spot was placed on the focal point of a 10x lens, the PI polymer film was placed on a stage, the XY moving and speed was precisely controlled by a computer with submicron resolution. The $\mathrm{T}$ shape of microchannel ( $200 \mu \mathrm{m}$ wide, $4 \mathrm{~cm}$ long and $50 \mu \mathrm{m}$ depth) with $1 \mathrm{~cm}$ of inlet microchannel were firstly photoablated on the PI film dynamically with a square metal mask (ArF-1 type), and then, the staggered herringbone (SH) pattern with 17 $\mu \mathrm{m}$ depth and total of 15 mixing cycles was grooved statically using the mask along the main microchannel with $2.07 \mathrm{~cm}$ long at the position of $1.5 \mathrm{~cm}$ from the inlet (ArF-SH type). In the meantime, for Y-shaped microchannels with $53 \mathrm{~cm}$ long $(200$ or $500 \mu \mathrm{m}$ wide, $50 \sim 60 \mu \mathrm{m}$ depth), the PI films were directly ablated by UV laser without mask. The laser spot size was fixed to become a $25 \mu \mathrm{m}$ diameter circle, and the stage was controlled to photoablate the film along the channel as designed (UV-1 type) or across the channel (UV-2 type) in a repeatedly scanning mode, respectively, and finally generating the line grooved microchannels. In particular, the staggered herringbone (SH) pattern with $\sim 5 \mu \mathrm{m}$ depth was also ablated along $9 \mathrm{~cm}$ long microchannel at the position of $0.5 \mathrm{~cm}$ from the inlet merging position except circular channel part. To analyze the different surface patterns of microchanel, the ArF-1 and UV-1 microchannels were measured using surface profiler Dektak-8 (VEECO, USA).

Mixing Efficiency of Microreactor. To evaluate the mixing efficiency of microfluidic devices with different surface patterns, the fluorescent distribution images were taken with a microscope Leica DMI3000B (Leica microsystems, Germany). In the case of the ArF-1 and ArF-SH microreactors, pure water was infused at a flow rate $4 \mu \mathrm{L} / \mathrm{min}$ through the inlet of the main microchannel, and an aqueous fluorescent solution with $250 \mu \mathrm{M}$ was provided from the other inlet of microchannel at flow rate $1 \mu \mathrm{L} / \mathrm{min}$. The fluorescent distribution images were taken at regular distance along the microchannel. The microreactor with staggered herringbone (SH) mixing pattern rendered completely homogeneous phase only after $0.28 \mathrm{~cm}$ flow along the mixer portion as shown in Figure $1 \mathrm{~b}$ ), it is clearly contrast to the fluorescent image in Figure 1 a) of microreactor with plain surface, which the significant mixing was observed after inflowing $3 \mathrm{~cm}$ from the inlet merging point.

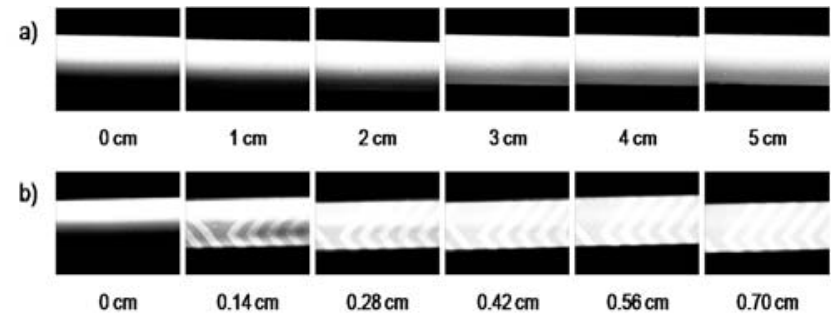

Figure 1S. Comparative fluorescent distribution images of a) ArF-1 and b) ArF-SH at various points along the channel

Tubing and Serial Connection of Film Microreactors. The film microreactors were connected to pumping devices via capillary tubes (Upchurch Scientific, USA). A sealing material Duralco-4525 (Cotronics corp., USA) was used to secure the connection between the in/outlet holes on the PI film and the standard tube connection (Nanoports, Upchurch Scientific). Alternatively, UV type film microreactors were fixed by a buckle type of aluminum chip holder (ICH-03, IMT, Japan) to connect the holes by using a screw type of Teflon ferrule with no use of adhesive materials. In addition, for Vilsmeier-Haack formylation reactions, two sheets of UV-2 film microreactors were serially connected by a capillary tube as seen in Figure 2S. To sweep up microchannel before and after reactions, solvents were infused very quickly into the microreactors by syringe pump then dried with nitrogen gas.

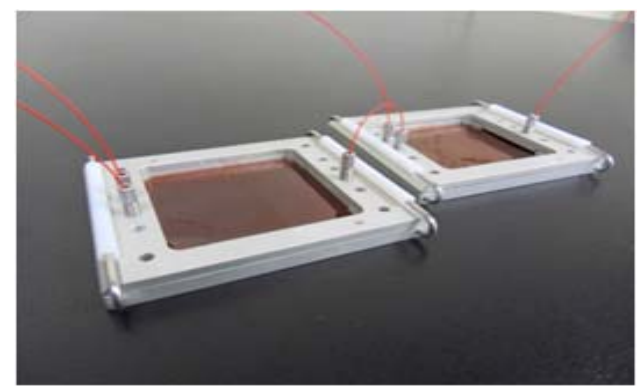

Figure 2S. Set of microreactors serially connected for Vilsmeier-Haack formylation reaction 


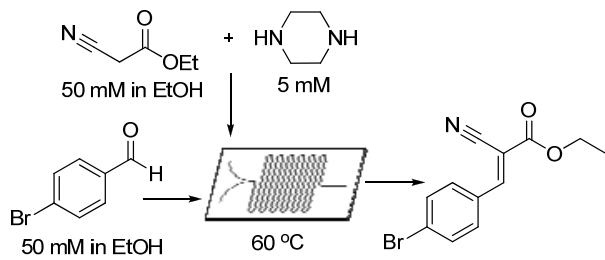

Scheme 1S. Knoevenagel condensation reaction in 3 different UV type microreactors.

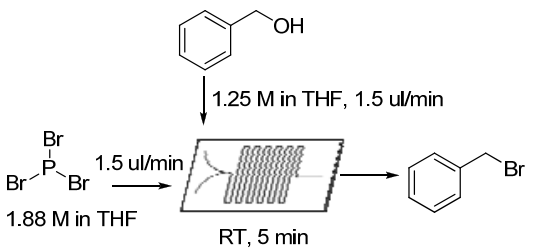

Scheme 2S. Bromination of benzylalcohol in UV-2 microreactor 